Rabaska

Revue d'ethnologie de l'Amérique française

\title{
Marius Barbeau, Le rossignol y chante, et nous et nous...
}

\section{Michèle Gardré-Valière et Michel Valière}

Volume 13, 2015

Présence de Marius Barbeau : l'invention du terrain en Amérique française. Autour d'un legs centenaire (1914-2014)

URI : https://id.erudit.org/iderudit/1033754ar

DOI : https://doi.org/10.7202/1033754ar

Aller au sommaire du numéro

Éditeur(s)

Société québécoise d'ethnologie

ISSN

1703-7433 (imprimé)

1916-7350 (numérique)

Découvrir la revue

Citer cet article

Gardré-Valière, M. \& Valière, M. (2015). Marius Barbeau, Le rossignol y chante, et nous et nous... Rabaska, 13, 112-123. https://doi.org/10.7202/1033754ar d'utilisation que vous pouvez consulter en ligne.

https://apropos.erudit.org/fr/usagers/politique-dutilisation/ 


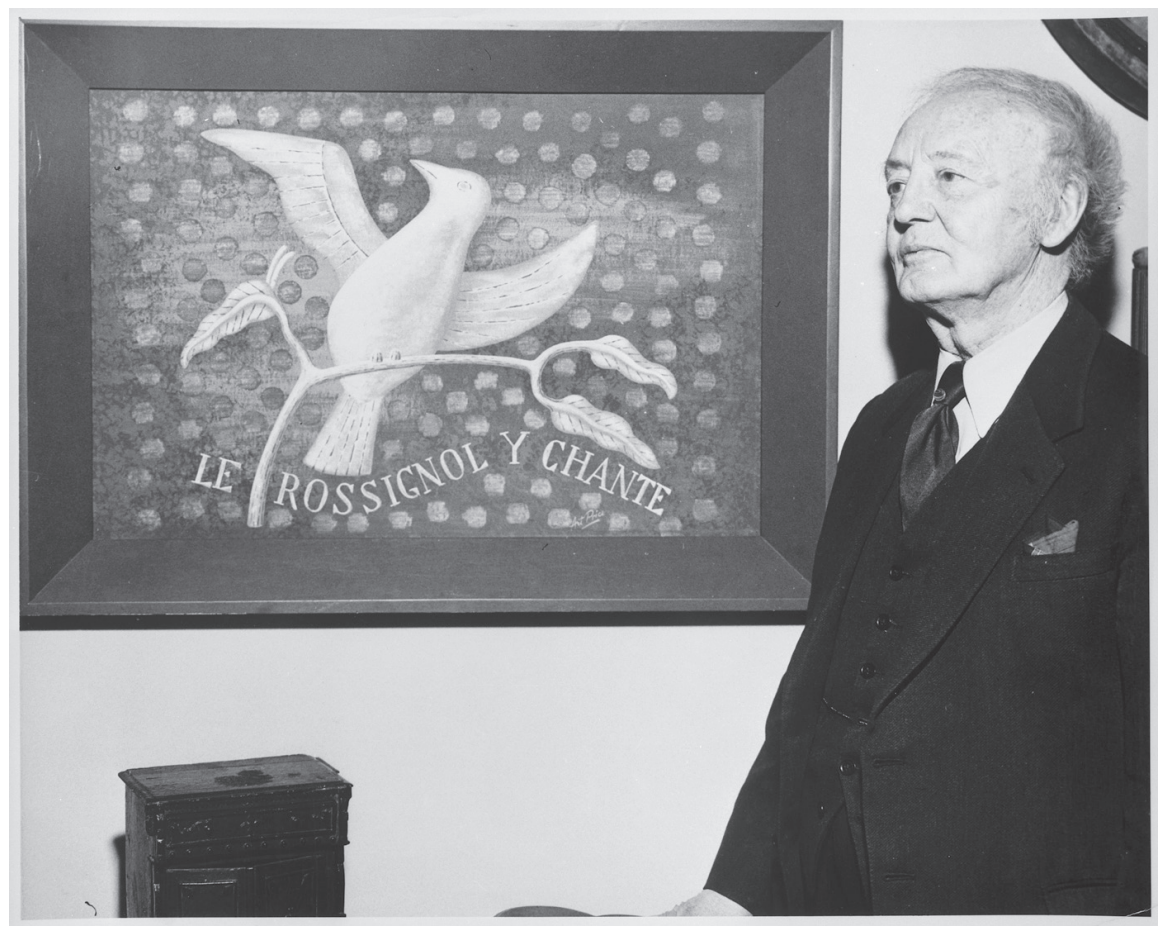

Marius Barbeau, avec le dessin Le rossignol y chante d'Arthur Price [1962 ou 1963].

Source : Musée canadien de l'histoire no 86-1267. 


\section{Marius Barbeau, Le rossignol y chante, et nous et nous...}

MichÈLE GARDRÉ-VALIÈRE

Association régionale pour la promotion de l'ethnologie Poitiers

Michel VALIÈRe

Ethnologue, membre associé au Laboratoire Mimmoc

Université de Poitiers

Alors jeunes chercheurs et enseignants isolés dans les Brandes du HautPoitou, éloignés des cercles universitaires ${ }^{1}$, nous étions surtout préoccupés dans la décennie 1960 d'éducation populaire de masse, dans l'esprit défini par le Gouvernement provisoire à la Libération de la France. Autant dire que nous ne connaissions Marius Barbeau et son œuvre que par des citations, des notes ou des références bibliographiques dans les travaux de Patrice Coirault et notamment dans Formation ${ }^{2}$. Mais Marius Barbeau ne prit corps à nos yeux ${ }^{3}$ qu'à la suite d'une rencontre à Toulouse avec l'ethnomuséographe du Musée national canadien, Madame Carmen Roy. Cette dame nous fit servir - cadeau de fée ! - Le rossignol y chante ${ }^{4}$ du prestigieux folkloriste, dont nous savons désormais que c'était le premier volet du futur triptyque Répertoire de la chanson folklorique française du Canada, avec En roulant ma boule (posthume, 1982); Le roi boit (id., 1987).

1. Ronald Labelle a bien souligné cette situation à la suite de l'une de ses visites en PoitouCharentes dans son article « Le Folklore musical en France : tendances actuelles dans la recherche et la diffusion ", Canadian folk music, Bulletin de musique folklorique canadienne, vol. 18, n 1, janv. 1984 : p. 9-11. Notons en outre que ce même numéro est illustré en couverture par une photo de Phil Thomas, présentant le guitariste Donald Deschênes accompagnant la chanteuse Charlotte Cormier, lors de «l'Assemblée générale à Edmonton [où] ils ont célébré Barbeau en chantant beaucoup de ses chansons ».

2. Patrice Coirault, Formation de nos chansons folkloriques, Paris, Scarabée, 1953-1963, 4 vol.

3. Ce n'est que beaucoup plus tard, en consultant en ligne les Archives de Radio-Canada que nous avons pu visionner un film très émouvant sur ce chercheur, réalisé en septembre 1964, et surtout l'entendre chanter et conter. Un enregistrement sonore nous permit d'écouter une évocation de la vie et de l'œuvre de M.B. par Luc Lacourcière, diffusée en 1970 (un an après sa mort).

4. Marius Barbeau, Le rossignol y chante : première partie du Répertoire de la chanson folklorique française au Canada, Ottawa, Ministère du Nord canadien et des ressources nationales et Musée national du Canada, 1962. 
Cette découverte conféra en conséquence un ancrage historique et géographique, en un mot humaniste, à nos propres collectes ${ }^{5}$ que nous jugions parfois anecdotiques, au gré de nos intuitions, œuvrant dans le sillage du recteur Léon Pineau ${ }^{6}$ ou de Jérôme Bujeaud ${ }^{7}$. En fait, nous les avions inscrites dans une perspective d' « ethnologie d'urgence» de la France ${ }^{8}$. La lecture du Rossignol et en particulier la conclusion de sa préface (p. 11) nous apporta la conviction d'une communauté d'esprit partagée avec l'auteur, notamment lorsqu'il cite Marguerite d'Harcourt :

Combien il est émouvant de retrouver là-bas, sur les rives du Saint-Laurent ou dans les villages de Gaspésie, des chansons normandes, poitevines, vendéennes ou saintongeaises qui se sont gardées pures, avec leur saveur intacte dans les mots et dans la musique, grâce à une tradition plus fidèle que celle de leur pays d'origine ![...]

Ainsi cet ouvrage, certes non représentatif à lui seul de la monumentale œuvre de Marius Barbeau, devint-il pour nous un portail virtuel entre les Français d'Amérique du Nord et les descendants de leurs ancêtres des « vieux pays », au sein desquels nous nous étions immergés, posture indispensable pour notre approche ethnographique que nous souhaitions holiste. Par ce truchement, depuis les deux bords de l'Atlantique Nord, des interprètes, des collecteurs, mais aussi des analystes et des classificateurs dont Patrice Coirault et Conrad Laforte, contribuaient-ils à écrire et régénérer tout un monumental Romancero : notre patrimoine culturel commun francophone. Il s'ensuivit un émerveillement, de multiples désirs d'échanges, de riches rencontres entre Québécois, Acadiens/Cajuns et des ressortissants du Centre-Ouest de la France, bref, un abaissement des distances, un rapprochement des époques par la mémoire commune des chansons 9 .

5. Cf. Catherine Robert, « Le Patrimoine culturel de l'Université de Poitiers, sa mise à disposition et sa valorisation : le fonds Valière », dans Rabaska, vol. 9, 2011, p. 236-240.

6. Deux ouvrages de cet ancien recteur de l'Académie de Poitiers nous ont sensibilisés très tôt, notamment en raison de la coextension des terrains d'enquête respectifs : Léon Pineau, Les Contes populaires du Poitou, Paris, Leroux, 1891 ; ou encore, Léon Pineau, Le Folk-lore du Poitou, Paris, Leroux, 1892.

7. Jérôme Bujeaud, Chants et chansons populaires des provinces de l'Ouest : Poitou, Saintonge,

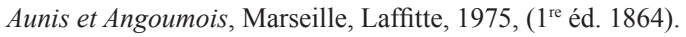

8. «Pour une ethnologie d'urgence de la France » : journées de la Société d'ethnologie française, Limoges, 25-27 octobre 1979, Ethnologia, n 17 à 20, 1981.

9. Patrice Coirault, Chansons françaises de tradition orale. 1900 textes et mélodies collectés par Patrice Coirault. Ouvrage révisé et complété par Marlène Belly et Georges Delarue, Paris, Bibliothèque nationale de France, 2013 ; Ernest Gagnon, Chansons populaires du Canada, Québec, Bureaux $\mathrm{du}$ « Foyer Canadien », 1865 ; Ronald Labelle (dir.), Chansons acadiennes de Pubnico et Grand-Étang tirées de la collection Helen Creighton, Darmouth, Helen Creighton Folklore Society et Moncton, Chaire de recherche McCain en ethnologie acadienne, 2008, (éd. bilingue, français-anglais) ; Conrad Laforte, Le Catalogue de la chanson folklorique française, Québec, Presses de l'Université Laval, 6 vol.,

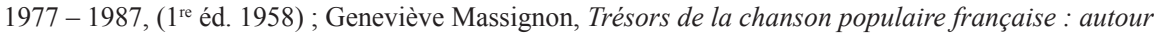
de 50 chansons recueillies en Acadie. Revu, corrigé et édité par Georges Delarue, Paris, Bibliothèque 


\section{La « fleur des chansons amoureuses $»^{10}$}

Déjà, le seul énoncé du titre de l'ouvrage de Marius Barbeau nous renvoyait quelques années auparavant à l'une de nos informatrices, chanteuse et conteuse très âgée. En effet, Marie Magnant, vêtue tout de noir, trace des multiples deuils de la première moitié du $\mathrm{Xx}^{\mathrm{e}}$ siècle français, nous avait littéralement subjugués, moins par ses qualités vocales et d'élocution dans un registre grave, que par son savoir oral et sa personnalité toute empreinte de vivacité et de spontanéité. Son répertoire dont nous avons rendu compte dans Mélanges posthumes autour de l'œuvre de Conrad Laforte ${ }^{11}$ recelait, entre autres, deux chansons où le « rossignol» occupait une place dans les deux incipits : "Rossignolet du bois joli »; "Rossignolet du bois, rossignolet sauvage ». Ce seul fait, qu'une vieille dame en deuil nous chante des chansons aux topiques printaniers, nous parut alors sinon comme un paradoxe, en tout cas comme une véritable antilogie (les linguistes préfèreront le terme oxymoron). Qu'à cela ne tienne! Aussi, riches de notre jeune expérience, la lecture de Marius Barbeau piqua au vif notre curiosité sans limites. En effet, le savant avait placé au troisième rang de son ouvrage ${ }^{12}$ Rossignolet $d u$ bois joli ${ }^{13}$. De notre côté, nous l'avions quelque peu « sacralisée » dans notre fonds, à la fois par la sensation de son ancienneté «Renaissance », par la poésie que nous voulions bien lui trouver, par l'émotion quasi stendhalienne, chaque fois renouvelée de Michèle qui l'interprétait ${ }^{14}$ et de Michel son « premier » auditeur-admirateur. Cette émotion, Marguerite et Raoul d'Harcourt, analystes et éditeurs de chansons recueillies par Marius Barbeau, nous semblèrent l'avoir éprouvée, qui écrivaient dans le commentaire faisant suite à ladite chanson, dans la version qu'ils avaient retenue, celle de Charles

nationale de France, 1994, 2 vol.

10. Nous empruntons ce titre de partie à celui de l'ouvrage éponyme publié en 1600 à Rouen chez Andrian de Launay, devant le Palais, au Compas d'or (cité par Jean-Baptiste Weckerlin, L'Ancienne chanson populaire en France (16 et $17^{e}$ siècle), Paris, Garnier, 1887, p. XXVII).

11. Michèle Gardré-Valière et Michel Valière, « Marie Magnant en Haut-Poitou : approche d'un répertoire chanté » dans Jean-Nicolas de Surmont (dir.), "M'amie, faites-moi un bouquet... ». Mélanges posthumes autour de l'œuvre de Conrad Laforte. Publiés avec la collaboration de Serge Gauthier, Québec, Presses de l'Université Laval, Éditions Charlevoix, coll. « Les Archives de folklore » 30, 2011, p. 273- 298.

12. En tête du premier chapitre « Oiseaux messagers d'amour », p. 23.

13. Cette chanson est classée sous le numéro 504 par Patrice Coirault, dans le tome 1 de son Répertoire des chansons françaises de tradition orale. Ouvrage révisé et complété par Georges Delarue, Yvette Fédoroff et Simone Wallon (vol. 1 et 2) ; Georges Delarue, Marlène Belly et Simone Wallon (vol. 3), Paris, Bibliothèque nationale de France, 1996-2006, 3 vol., sous le titre générique « Le rossignolet qui invite à changer d'ami ». De son côté, Conrad Laforte l'inscrit sous le code II, E-51, sous le titre «Le printemps les filles changent d'amant", dans son Catalogue de la chanson folklorique française, Laval, Presses de l’Université Laval, vol. 2, 1981.

14. Par habitude, Michèle s'exerçait à apprendre les chansons recueillies par nous en faisant dérouler le ruban magnétique original ; mais, dans le cas présent de Marie Magnant qui était dotée d'une voix très grave, Michèle devait pour chanter en même temps qu'elle placer sa voix à l'octave supérieure. 
Barbeau, père du collecteur : «Bien placée dans une jolie voix, c'est un régal que d'entendre cette mélodie, touchante et fraîche. ${ }^{15}$ "

À y regarder de plus près, le rapprochement des deux éditions de « Rossignolet du bois joli », chanson $n^{\circ} 1281$ du même informateur de Beauce, Charles Barbeau, dans Le rossignol y chante (p. 23) comme dans l'ouvrage des d'Harcourt (p. 154) laisse apparaître des différences notoires tant en matière musicale qu'au plan de la coupe et donc du texte. Marius Barbeau, par son approche archétypale à partir de « quatre versions recueillies aux environs de Québec et en Cadie », propose « un texte critique » de trois strophes. Il s'est contenté de la structure musicale du premier couplet, inférant que les autres strophes devaient se chanter à l'identique, s'éloignant, avec sa forme rectifiée, de ses sources informatives initiales conservées au Musée national canadien à Ottawa. En revanche, Marguerite d'Harcourt a soigneusement noté les mélodies des deux premiers couplets qui présentent des différences notables telles que nuances dans la coupe ou changement de rythme. Ce faisant, elle invite à interpréter le troisième couplet « sur la musique du premier ». Ainsi celle-ci a-t-elle adopté au fil de l'ouvrage une posture que nous qualifierons de «plus moderne », puisqu'elle tend à restituer, au plus près, la variabilité de la musique et du texte, au sein de chaque item, attitude plus respectueuse de l'oral, comme nous l'entendons, nous, aujourd'hui.

En fait, nous devons comprendre que Marius Barbeau a reconstruit une version canonique sur un seul et même air, plus lisse, motivé en cela par la nécessité affichée de la fournir aux artistes des « deux festivals de la Chanson donnés au Château Frontenac à Québec, en 1927-1928 », comme il a tenu à le préciser dans sa préface (p. 6). Il s'oppose ainsi au choix des d'Harcourt et peut-être donc à la reconnaissance d'une esthétique mélodique particulière, celle-là même qui avait fondé notre désir d'une découverte plus approfondie de son œuvre. Toutefois, nous ne nous risquerons pas dans les analyses musicologiques à la recherche de topiques musicaux ou de traits justifiant nos émotions ; pourtant, ce sont bien elles qui nous motivaient alors dans notre progression sur le terrain et la poursuite de notre recherche assidue. Aussi nous nous sentions en sympathie avec le collecteur, dont on sait qu'il fut disciple à Oxford de l'anthropologue et juriste, Robert Ranulph Marett (1866-1943) et qu'il fut également inspiré par les travaux et l'œuvre de l'anthropologue allemand émigré aux États-Unis, Franz Boas (1858-1942), l'un des pionniers de l'enquête de terrain, initiée dans le Nord canadien ${ }^{16}$. C'est grâce à

15. Marguerite et Raoul d'Harcourt, Chansons folkloriques françaises au Canada : leur langue musicale, Québec, Presses universitaires Laval, Paris, Puf, 1956, p. 155.

16. D'après Gérald Gaillard, Dictionnaire des ethnologues et des anthropologues, Paris, A. Colin, 1997 ; ainsi que Gamila Morcos et al., Dictionnaire des artistes et des auteurs francophones de l'Ouest canadien, Québec, Presses de l’Université de Laval, 1998. 
celui-ci qu'il étendit ses premières recherches sur les Amérindiens aux contes et chansons du Canada francophone, dans le dessein, suggéré par F. Boas, d'évaluer, au dire de Luc Lacourcière ${ }^{17}$, d'éventuelles influences de l'apport colonial français sur les répertoires indiens.

Marius Barbeau, fin observateur minutieux et efficace des « créations culturelles » sur le terrain, nous avait donc conquis dès la première lecture. Pourtant, ce sont davantage leurs exégètes, les époux d'Harcourt, musicologues français, qui confortèrent notre point de vue en mettant en exergue la richesse musicale de ce fonds, du moins de l'échantillon de deux cent quarante chansons portant sur cent quatre-vingt-six sujets distincts qu'ils avaient pris en compte, parmi les mille qu'ils avaient transcrites, et dont le choix « avait été surtout déterminé par des raisons d'esthétique ou des particularités musicales dignes d'être fixées ${ }^{18} »$.

\section{Chansons en écho}

Par-delà l'extrême sympathie à nos yeux du titre de l'ouvrage, c'est davantage la parenté patente entre nombre d'éléments de nos corpus respectifs qui nous stimula. Rapprochons donc quelques poésies populaires que nous aimons et que nous semblons partager avec l'auteur. Ainsi, à titre d'exemple, la chanson page 397 « Derrière chez nous yat [sic] un bateau », recueillie en 1922 à PortDaniel (Baie des Chaleurs), figure avec quelques nuances (même thématique, même coupe, timbres différenciés) dans notre propre corpus sous l'incipit « Sur la rivière de Bordeaux », recueillie en 1966 à Blanzay (Vienne) :

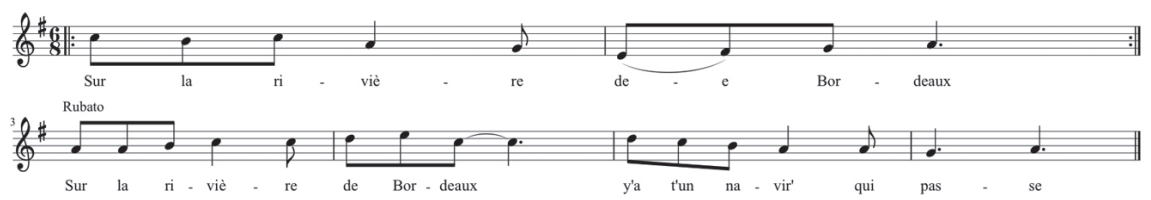

Sur la rivière de Bordeaux (bis)

Sur la rivière de Bordeaux, y a-t-un navire qui passe

Le matelot qui est dedans (bis)

Le matelot qui est dedans, grand Dieu qu'il est malade

Toutes les dames de Bordeaux (bis)

Toutes les dames de Bordeaux, ont bien été le voir-e

$\mathrm{Y}$ a rien que la jeune Isabeau (bis)

$\mathrm{Y}$ a rien que la jeune Isabeau, que son père la garde

Il ne l'a point toujours gardée (bis)

Il ne l'a point toujours gardée, elle a été le voir-e

- Oh matelot, beau matelot (bis)

17. Cf. supra, note 3 .

18. Marguerite et Raoul d'Harcourt, op. cit., 1956, p. 55. 
Oh matelot, beau matelot, mangerais-tu une poire?

- Oh oui, oh oui, jeune Isabeau (bis)

Oh oui, oh oui, jeune Isabeau, venant de ta personne

En la coupant, en la parant (bis)

En la coupant, en la parant, le navire y décampe

N'y fiyant pas cent lieues sur l'eau (bis)

N'y fiyant pas cent lieues sur l'eau, que la belle s'y chagrine

- Qu'avez, qu'avez jeune Isabeau (bis)

Qu'avez, qu'avez jeune Isabeau, qu'avez qui vous chagrine

- Mon père, ma mère m'avaient toujours dit

Mon père, ma mère m'avaient toujours bien dit

Mon père, ma mère m'avaient toujours dit que je serai fille perdue

- Ne pleurez point jeune Isabeau (bis)

Vous passerez la nuit avec moi, le jour avec ma mère (bis).

Cette chanson est classée par Coirault dans la rubrique « Rapts » sous le titre « L'Amant qui feint d'être malade », cote 1312, mais, apparemment et sauf erreur, " oubliée » par Laforte dans son catalogue. Dans le volume 1 du Répertoire, deux versions seulement sont signalées : l'une de Damase Arbaud, en langue provençale mais sans mélodie associée, l'autre de Marius Barbeau, en français. On comprendra qu'avec notre version, la deuxième en français, à l'époque de nos collectes, nous ayons pu concevoir un lien entre nos textes et, par extension, entre nos corpus. Depuis, grâce au précieux travail de Marlène Belly ${ }^{19}$ et de Georges Delarue, dans la foulée de $\uparrow$ Simone Wallon, trois autres versions ont été adjointes dans le volume 3 du Répertoire.

Nous ne nous arrêterons que très peu sur des chansons trop célèbres comme « Cherchant ses amours ${ }^{20}$ » représentées dans l'ouvrage par trois versions (p. 181-186), mais dont nous avons également recueilli trois versions dont la première en 1965 à Sommières-du-Clain (Vienne) sous l'incipit « Jeune militaire revenant de guerre, cherchant ses amours », la deuxième en 1969, la troisième en 1982 à Usson-du-Poitou.

Rien d'étonnant non plus à trouver dans Le Rossignol un texte critique établi à partir de trois versions de la chanson de la pauvre Hélène, « Sur le

19. Marlène Belly, éditrice scientifique des chansons de Coirault (cf. supra, note 13) est l'auteur de nombreux travaux. Au nombre de ceux-ci : «Le Miracle de la muette : un air, un timbre, une coupe », Autour de l'œuvre de Patrice Coirault, actes du colloque organisé par le Département de musicologie de l’Université de Poitiers, les 24 et 25 nov. 1994, Parthenay, FAmDT, 1997, p. 84-98. Retenons encore: Marlène Belly, Variations dans la chanson de tradition orale française ; étude d'une pièce : La bergère muette, DÉA, sous la direction de Nicole Belmont, Paris, ÉHÉss, 1993, vol. 1, 100 p., Recueil annexe, vol. 2, 123 p. (multicopié).

20. Coirault dans Formation a consacré dix pages d'étude à ce type " Brave capitaine " et l'a classée dans son Répertoire sous le n 1425, et Laforte, dans son Catalogue: II, A-75 (sous le titre « La Fille du Maréchal de France »). 
Pont de Londres » (p. 209-211). Les jeunes Français du Centre-Ouest ont chanté, eux, les malheurs correspondants d'Adèle (ou d'Hélène), sous le titre « Sur le Pont du Nord ${ }^{21}$ », parfois « Sur le Pont de Nantes ».

On pourrait passer ainsi en revue les rondes ou jeux dansés comparables. Par exemple, " Dans la Cadie luy a » chanté en 1916 par Louis Simard 1'Aveugle à Saint Irénée qui en connaissait deux mélodies (Le Rossignol, p. 227). L'une d'elles, qu'il tenait encore enfant de ses sœurs, laisse à comprendre qu'il s'agit d'un jeu dramatique d'enfants que nous pourrions corréler avec une ronde mimée de filles de notre propre fonds, «Derrière les lilas blancs l'y a-t-une fontaine ${ }^{22} »$, enregistrée (v. 1971) auprès d'une jeune fille de la Basse Touche, commune de Magné (Vienne). Nous aurions aimé pouvoir confronter, s'il l'avait donné, avec celui de M. Barbeau, le déroulement de ce jeu où l'une des trois fillettes enfermées avec « le petit roi d'Angleterre » dans le cercle d'enfants, vexée de ne pas avoir été l'objet de son choix, lui lance un défi : «Je te ferai la guerre » puis elle mime son meurtre, perpétré avec une quenouillette, emblème féminin de l'Ancien temps, celui-là même qu'arborait la Pucelle de Domrémy, notre héroïne nationale, Jeanne d'Arc. Nous ne gloserons pas davantage ici ce désir d'en finir avec l'ennemi traditionnel historique du Royaume de France ainsi mis en scène.

Au total, une vingtaine de chansons représentées dans le Rossignol auraient ainsi pu faire l'objet de rapprochements pertinents (beaucoup plus si l'on avait pris en compte les deux volumes suivants que nous avons découverts depuis !) ; mais le temps et l'espace impartis ici ne nous le permettent pas et nous souhaitons que de futures rencontres y soient plus propices. Toutefois cette parenté ressentie entre nos ensembles en présence n'est qu'illusoire compte tenu des volumes d'éléments respectifs. En effet, les realia des terrains américains et celles de nos « vieux pays » n'auront pas motivé les chanteurs comme leurs collecteurs de la même façon. Le Rossignol nous aura de ce fait même encouragés par exemple à rechercher davantage dans notre aire des « complaintes anecdotiques » liées à des événements locaux dramatiques ${ }^{23}$. C'est aussi la richesse des collectes de Barbeau, évaluées par nous à l'aune du premier volume reçu en cadeau du Canada qui nous incita à échanger avec

21. Coirault, Répertoire : $\mathrm{n}^{\circ} 1725$; Laforte, Catalogue : I, B-2 (sous le titre « Danseuse noyée »).

22. Coirault, Répertoire : $\mathrm{n}^{\circ} 3809$; Laforte, Catalogue: I, C-1 (sous le titre « Les Trois filles et le Roi d'Angleterre »). De son côté, G. Massignon (op. cit., 1994 : p. 83-87), a recueilli plusieurs versions en Acadie et dans l'Ouest de la France, précisant elle aussi qu'il s'agit d'une ronde de fillettes qu'elle présente sous l'incipit « Derrière les lilas blancs, il y a-t-une fontaine », $\mathrm{n}^{\circ} 13$ du corpus édité sous le titre générique « Le petit roi d'Angleterre ».

23. Michel Valière, "Chansons de colportage et chansons de circonstances recueillies en Poitou », Bulletin de la Société des antiquaires de l'Ouest, $4^{\mathrm{e}}$ série, t. xv, $2^{\mathrm{e}}$ trim. 1977, p. 103-142. 
nos homologues québécois ou acadiens ${ }^{24}$, dont l'ethnomusicologue Charlotte Cormier $^{25}$.

\section{Charlotte : ses chansons, son terrain}

Une nouvelle rencontre nous permit une approche à la fois personnelle et collective des Acadiens qui ont partie liée avec la région de PoitouCharentes où nous nous sommes beaucoup investis, vu l'histoire de l'émigration généralement mise en avant au titre de la mémoire et du souvenir. L'épopée acadienne ${ }^{26}$, la «complainte acadienne », nous les avons ainsi découvertes au travers de l'une de nos collègues, passionnée tout autant que nous de chansons de tradition orale et, plus largement, d'ethnomusicologie. Qu'un peuple qui a tant souffert de sa disgrâce, de son exil, de son déni trois siècles durant s'exprime dans notre langue commune donnée en partage au sein de la francophonie semble relever du miracle historique. Que, depuis un siècle et demi, des artistes, des ecclésiastiques, des gens de lettres se soient attachés à recueillir et à sauvegarder comme un bien précieux, comme de vraies reliques, et à diffuser des « trésors » de littérature populaire force à l'admiration et ne laisse toujours pas de nous étonner.

Aussi la rencontre avec une jeune folkloriste acadienne encore dans son parcours universitaire, Charlotte Cormier, deviendra-t-elle pour nous, là encore, une source d'émotion, prémices à de nouvelles réflexions sur la profondeur historique des textes chantés que nous accumulions au cours de nos prospections en Haut-Poitou. Ceux-ci nous voulions légitimement les inscrire dans le prolongement direct des travaux d'éminents prédécesseurs. Nos réflexions devaient s'orienter également sur la dispersion spatiale des chansons, leurs variations subséquentes ainsi que leurs fonctions sociales réelles ou supposées, voire leur interprétation.

À l'origine de ces échanges avec l'amie Charlotte Cormier, alors jeune chercheuse et enseignante à l'Université de Moncton (Nouveau-Brunswick), un élu départemental qu'elle avait rencontré dans le cadre de manifestations de jumelage entre cités et qui l'orienta, en la recommandant, dans notre direction. En effet, en quête de sources chantées de France pour nourrir ses études acadiennes sur la chanson, et peu satisfaite de l'accueil que lui avaient réservé

24. Citons pour l'exemple Georges Arsenault, auteur de La Chandeleur en Acadie, TracadieSheila, La Grande Marée, 2011, ainsi que maints articles et ouvrages sur les traditions et la chanson. Nous n'oublions pas non plus Lauraine Léger ; Barry Ancelet ; la linguiste Ginette Richard ; et tant d'autres amis.

25. On lui doit entre autres : Charlotte Cormier et Donald Deschênes, « Joseph-Thomas LeBlanc et le romancero inachevé », dans Folklore canadien, vol. 13, n 2, 1991, p. 55-66 ; Charlotte Cormier, « La Musique traditionnelle en Acadie ", Mémoires de la Société royale du Canada, quatrième série, t. xv, 1977, p. 239-259.

26. Michel Valière, "Mobilisations identitaires d'Acadiens ", dans Les Cahiers européens des sciences sociales, $\mathrm{n}^{\circ}$ 6, 2014 : (Dossier « Minorités et Intégration »), p. 73-84. 
à Paris des responsables d'une institution nationale pourtant appropriée en matière d'ethnomusicologie, c'est donc vers le Poitou et les collections que nous avions rassemblées à titre personnel ou dans un cadre plus large, avec l'association Centre culturel : La Marchoise, à Gençay (Vienne), qu'elle souhaita se tourner dans les années 1970. Ainsi en s'installant pour une période d'un mois à notre domicile, suivie d'autres visites ultérieures dans d'autres familles, initiait-elle à sa façon une situation d'ethnologie participante.

Cette impression se forgea à partir de la prise de conscience de la corrélation de plusieurs patronymes d'Acadie et de quelques-uns du PoitouCharentes, en particulier du sien propre. D'un autre point de vue, lexicographique, elle saisit au vol des mots partagés, tels "éloise » dans le sens d' « éclair », « abrier » pour « couvrir », « basir », pour « mourir » ou « disparaître », « éparer » pour « étaler, étendre », ou « garocher» pour « jeter, lancer », sans parler des «aboiteaux», ces « levées de terre » aussi indispensables dans les marais acadiens que dans ceux du Golfe des Pictons. Pour ce qui est des traits morphologiques entendus et qui lui parlaient aussi du pays, en fait, si l'on se réfère à l'ethnolinguiste Louise Péronnet, il semblerait que la grande majorité des traits acadiens « proviennent du français archaïque et populaire plutôt que d'une région particulière de l'Ouest de la France ${ }^{27} »$. Nous devions partager avec elle cette impression tant il nous sembla reconnaître, à travers elle et la voix enregistrée de l'une de ses informatrices acadiennes, le parler d'aïeux maternels du marais de la Boutonne en Charente-Maritime, ou de quelques-unes de nos « informatrices privilégiées ${ }^{28}$ » du Sud de la Vienne.

La situation était donc propice à une confrontation amicale des répertoires de chants acadiens et de ceux que nous avions appréhendés dans le Civraisien et le Montmorillonnais d'autant que nous savions que le corpus engrangé, aux côtés d'autres, était considéré comme d'utiles sources orales par et pour les communautés francophones d'Amérique du Nord (Acadie ; Louisiane ; Québec) en quête de leurs origines identitaires. Une situation d'échanges dynamiques? de textes de la mémoire acadienne et de celle des « vieux pays » se faisant écho en dépit d'une histoire distincte, dans le dessein d'examiner quelques éléments nés de la contrainte des événements subis par les populations victimes d'aléas, fondateurs d'une situation « diasporique ».

Mais d'abord intéressons-nous à l'ouvrage de Charlotte Cormier, Écoutez tous, petits et grands ${ }^{29}$, accompagné de ses deux disques vinyles, qui rend

27. Louise Péronnet, "Le Français acadien », dans P. Gauthier et T. Lavoie (dir.), Français de France et Français du Canada. Les parlers de l'Ouest de la France, du Québec et de l'Acadie, Lyon, Université de Lyon III, Centre d'études linguistiques Jacques Goudet, 1995, p. 419 ; p. 399-439.

28. Nous empruntons ce syntagme à Michel et Françoise Panoff, L'Ethnologue et son ombre, Paris, Payot, 1968, p. 47 et passim.

29. Charlotte Cormier, Écoutez tous, petits et grands, Moncton, Les Éditions d'Acadie, 1978, (accompagné de ses deux disques vinyles $17 \mathrm{~cm} ., 33$ t.1/3). 
compte de son approche ethnographique dans le village de Pré d'en-haut qui fait partie de Memramcook, « la plus ancienne paroisse acadienne $»^{30}$. Notons en premier lieu l'élégance de ce petit ouvrage dont la couverture a été illustrée par la reproduction d'une huile sur toile de Jean-Claude Dupont, datée de 1977 et intitulée « La Chanteuse de complaintes », tout à fait appropriée au propos de l'ouvrage. En effet, Charlotte Cormier a rassemblé onze chansons de cinq chanteuses. Pas de chanteurs! Par petites touches, dans une approche impressionniste, on cerne progressivement la vie quotidienne des habitants de Beaumont, de Belliveau-Village, de Pierre-à-Michel, de Pré-d'en-haut. Toutes les chansons du livre et des deux vinyles, à des degrés divers, sont émouvantes de par l'authenticité des voix des chanteuses. Arrêtons-nous sur l'une d'entre elles, « La Fille du cavalier ${ }^{31}$ », dans la mesure où elle figure sous le titre « La Fille du geôlier » dans le Rossignol ${ }^{32}$, mais également dans notre propre fonds, à quelques nuances près, conformément au résumé qu'en fait Patrice Coirault dans le Répertoire, sous le numéro 1403 avec pour titre " Pierre et Françoise : La Fille du geôlier amoureuse d'un prisonnier ».

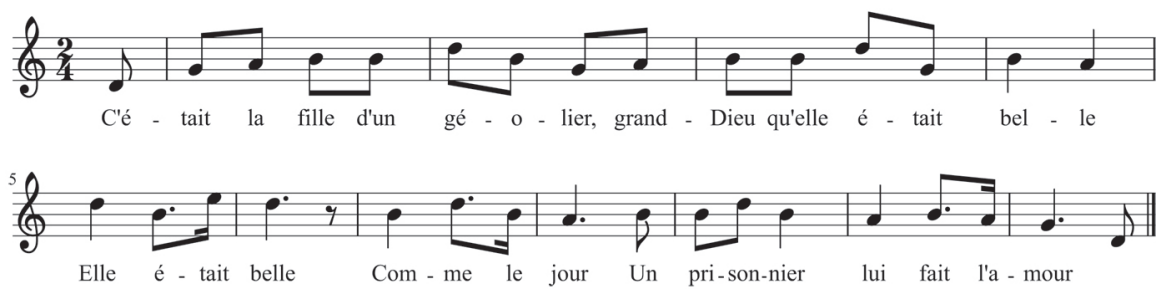

C'était la fille d'un geôlier grand dieu qu'elle était belle

Elle était belle comme le jour un prisonnier lui fait l'amour

De grand matin elle s'y leva s'en va trouver le juge

À ses genoux elle s'y jeta ayez pitié d'un pauvre soldat

Y a point de pitié pour ce soldat il est jugé il en mourra

Dessous le traversin du lit les clefs de la prison elle prit

À son amant ouvrit la porte

- Amant sortez de la prison les clefs elles sont à l'abandon

- De la prison j'en sortirai ma charmante maîtresse

Quand mon procès sera jugé de la prison j'en sortirai

Ils s'assièrent dessur un banc causèrent tous deux ensemble

L'amant regarde derrière lui le grand bourreau il vit venir

- Prenez l'anneau que j'ai au doigt et faites un autre amant que moi

- Un autre amant je n'en veux point je veux toujours le même

Mourir ici mourir là-bas je veux mourir entre tes bras

30. Ibid., p. 11.

31. Ibid., p. 56-57.

32. Barbeau, op. cit., 1962, p. 203-205. 
Quand il y fut sur l'échafaud la belle fondait en larmes

En arrachant ses noirs cheveux en regardant son amoureux

Les juges se dirent les uns les autres - Grand Dieu quel amour tendre

Laissez-les donc s'y marier tous deux ces deux jeunes et tendres amoureux ${ }^{33}$

Dans le deuxième volume du Catalogue de Laforte, «Pierre et Françoise » prend place sous la cote II, C-5. Marius Barbeau, parlant de ces « belles amours » voudrait bien les resituer dans une époque historique tout à fait remarquable : «[...] la fraîcheur et la simplicité du récit les place dans la meilleure période avant la contamination par l'imprimerie. » Nous n'avons pas recueilli l'avis de Charlotte Cormier sur ce point. Mais l'hypothèse de Marius Barbeau semble étayée par les écrits d'un historien médiéviste, Roger Vaultier : « [...] ceci n'est pas du domaine de la légende, un homme conduit à la potence pouvait, s'il était réclamé par une jeune fille, être délivré, c'était la rémission par mariage..$^{34} \gg$ Ajoutons encore que Montaigne ${ }^{35}$, philosophe et moraliste de la Renaissance, fait aussi allusion dans ses essais à cette tradition, toutefois sur un mode ironique. Aussi, réservons-nous notre avis sur cette fort belle histoire chantée, dont nous possédons encore une seconde version poitevine ${ }^{36}$ un peu plus concise, mais encore plus tendre. Ainsi va la vie, tant dans la virtualité des poésies chantées que dans la cruelle réalité humaine...

Ce qui n'aurait pu rester chez nous que lettre morte, futilité ou activité de loisirs, " collecter les chansons anciennes », se transforma ainsi en archives orales historiques, témoignages directs émouvants, tous propices à des confrontations entre nos répertoires réciproques, à l'occasion de belles « rencontres » à l'instar de celle que nous vivons ces jours-ci à Saint-Irénée, au Québec, la Belle Province.

Dans une autre approche, nous pourrions dire que, d'une certaine manière, nous avons tenté de « mesurer » l'impact pour nous et sur nous d'un ouvragephare de Marius Barbeau comme, pour la communauté des chercheurs rassemblés ici, l'influence de l'ensemble de son œuvre. C'est donc ce tout petit pas de bibliométrie, que nous offrons en hommage à tous les collecteurs et passionnés, proches ou lointains, et surtout à la mémoire de notre « Grand Ancien $^{37} », 1$ 'heureux auteur du Rossignol y chante.

33. Chantée en 1969 par $\uparrow \mathrm{M}^{\mathrm{me}}$ Augereau, dans le canton de Gençay (Vienne), avec pour incipit "C'était la fille d'un geôlier ». Partition établie par Michèle Gardré-Valière et Séverin Valière, musicien.

34. Roger Vaultier, Le Folklore pendant la guerre de Cent ans d'après les Lettres de Rémission du Trésor des Chartes, Paris, Librairie Guénégaud, 1965, p. Iv.

35. Montaigne, Les Essais, livre I, ch. XIV, Paris, PuF, « Quadrige », 1999, p. 51-52.

36. Chantée en 1970 par $\dagger M^{\text {me }}$ Pierron, du même canton : « La Fille du geôlier ».

37. Nous empruntons cet appellatif au folklore des étudiants de Poitiers de notre génération. 